\title{
Studies of the Aging Properties of the Mu2e Cosmic Ray Veto System
}

\author{
P. J. Farris*, R. C. Group, Y. Oksuzian \\ University of Virginia \\ E-mail: rcg6p@virgina.edu
}

\begin{abstract}
The Muon-to-Electron Conversion experiment (Mu2e) will operate at a single event sensitivity of $3 \cdot 10^{-17}$ with a total background of less than 1 event over the three-year lifetime of the experiment. To achieve this level of background, the Cosmic Ray Veto detector (CRV) will surround the Mu2e apparatus to veto penetrating particles that cause background. The CRV is required to have a detection efficiency of $99.99 \%$ throughout the lifetime of the Mu2e experiment. The CRV is comprised of extruded polystyrene scintillating strips and fiber which degrade over time, decreasing the efficiency of the CRV. Using a standard accelerated aging technique, several scintillator and fiber samples were heated to increase their rate of degradation. The results of these studies and the impact of aging on the CRV are presented.
\end{abstract}

The 20th International Workshop on Neutrinos (NuFact2018)

12-18 August 2018

Blacksburg, Virginia

${ }^{*}$ Speaker. 


\section{Introduction}

The Muon-to-Electron Conversion experiment (Mu2e) seeks to find physics beyond the Standard Model: the direct conversion of a muon to an electron without the emission of a neutrino $[1,2]$. Cosmic ray muons can interact with the material in the experiment and produce electrons that can mimic the desired signal. A detector called the Cosmic Ray Veto (CRV) will surround the Mu2e experiment and veto these backgrounds with an efficiency of $99.99 \%$.

A main component of the CRV is the dicounter, which is made by epoxying together two polystyrene scintillation counters. Each dicounter is then outfitted with optical fibers (Kuraray double-clad Y11, non-Stype [3, 4]) and instrumented with Hamamatsu silicon photomultipliers (SiPMs, model S13360-2050VE, 1584 pixels [5]). The performance of CRV prototype counters was measured in a test beam at Fermi National Laboratory [6]. Natural degradation of the polystyrene scintillating counters and fibers will decrease the efficiency of the CRV throughout the experiment's three-year lifetime. The current aging estimate for the CRV is $30 \%$ after ten years. To determine if the required efficiency is maintained throughout the lifetime of the experiment, the aging properties of the dicounters and optical fiber were investigated.

\section{Accelerated Aging}

Accelerated aging is a common technique to study the degradation properties of many materials. Polystyrene degrades faster at higher temperatures; thus, aging of polystyrene samples can be accelerated by placing them inside an oven. One sample is kept at a reference temperature to calculate the effective aging of the heated samples relative to the reference sample.

\subsection{Arrhenius Equation}

The Arrhenius equation [7] was used to model the reaction rate "constant" as a function of temperature. The ratio of the same reaction at two different temperatures simplifies to a ratio of two reaction rate constants. This ratio was used as the relative aging factor to calculate the effective aging time:

$$
\frac{k\left(T_{\text {high }}\right)}{k\left(T_{\text {ref }}\right)}=e^{\frac{E_{a}}{R}\left(\frac{1}{T_{\text {ref }}}-\frac{1}{T_{\text {high }}}\right)},
$$

where $k(T)$ is the reaction rate, $E_{a}=91 \mathrm{~kJ} / \mathrm{mol}$ [8] is the activation energy for the reaction, $T_{r e f}=23 \mathrm{C}, T_{\text {high }}=50 \mathrm{C}$, and $R$ is the universal gas constant. For this study, $E_{a}$ is the activation energy for the reaction between polystyrene and air.

\subsection{Configuration}

Three sets of dicounters were fabricated in June 2016 for the study: unassembled, 12 dicounter samples with no outfittings; assembled, 6 outfitted dicounter samples; special mix, 6 dicounter samples mixed with a higher percentage of $\mathrm{TiO} 2$ in the coating. Twelve spools of fiber were also tested. All test samples were placed in the oven in August 2016. The 12 sample sets were removed in 4-week intervals; 6 sample sets were removed in 8-week intervals. This provided several data 
points with which to model the aging. Additionally, one control sample for each set was stored outside the oven.

The dicounter samples were measured by placing them inside a light-tight box to reduce background noise. A Cs-137 source was placed on top of the box to induce scintillation, producing an output current. All samples were measured on the same day using the same removable fiber-SiPM jig. These methods ensured that aging of the measurement apparatus had no effect on the results.

The fiber samples were measured using a rotating drum to measure the light yield as a function of distance [4]. The light gain was measured using a photodiode outputting ADC counts. These samples were also measured in a two-day interval to ensure a negligible amount of aging during measurement.

\section{Results \& Conclusions}

After measurement, samples were normalized to their initial values and plotted against the effective aging time. The dicounter aging fit a double exponential indicating both fast and slow aging components. The relative errors are estimated to be $\sim \pm 1 \%$. For $E_{a}=91 \mathrm{~kJ} / \mathrm{mol}$, the aging after 10 years is $22 \%$. The special mix dicounters had the best performance while the assembled dicounters had the worst performance, as expected (see Figure 1, left). The fiber aging fit a single exponential and is trivially small: less than $1 \%$ per year. Thus, based on these studies, extrusion aging will be the dominant factor in the aging of the CRV.

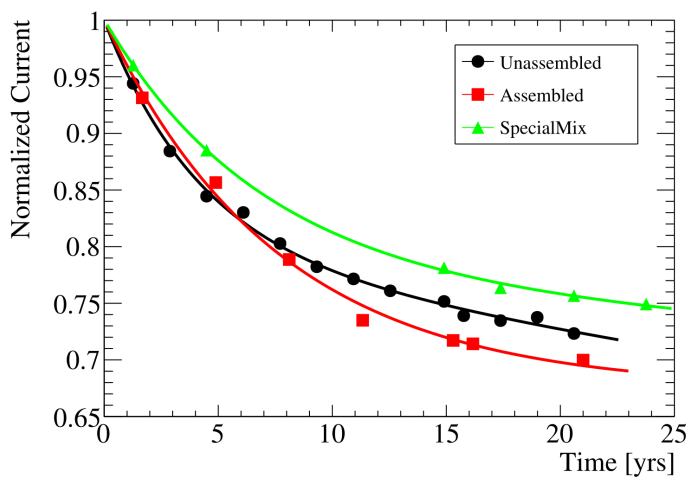

Figure 1: Dicounter aging.

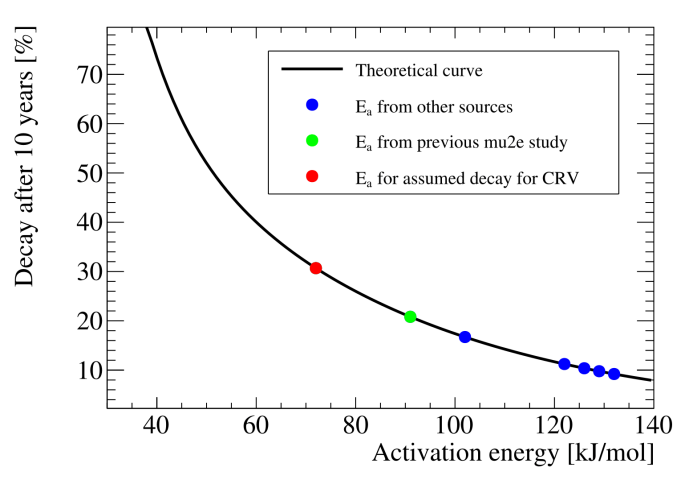

Figure 2: Aging at ten years as a function of $E_{a}$.

The activation energy, however, is not well known. The literature contains several values that range from 85 to $120 \mathrm{~kJ} / \mathrm{mol}[9,10]$ for the activation energy of polystyrene. Figure 2, right, shows a theory prediction generated by calculating the amount aged after 10 years for several values of $E_{a}$. Furthermore, it should be noted that non-accelerated aging and recent test-beam results point to a larger aging effect. It is possible that the Arrhenius equation does not encapsulate all of the effects that reduce dicounter performance with time. Additional studies on non-accelerated aging to check these results are ongoing. 


\section{References}

[1] L. Bartoszek et al. [Mu2e Collaboration], "Mu2e Technical Design Report," arXiv:1501.05241.

[2] R. H. Bernstein and P. S. Cooper, "Charged Lepton Flavor Violation: An Experimenter's Guide," Phys. Rept. 532, 27 (2013).

[3] Kuraray America, Inc., 00 Park Ave., NY 10166 USA; 3-1-6, NIHONBASHI, CHUO-KU, TOKYO 103-8254, JAPAN.

[4] E. C. Dukes, P. J. Farris, R. C. Group, T. Lam, D. Shooltz and Y. Oksuzian, arXiv:1811.04874, to be published in JINST.

[5] Hamamatsu Photonics K.K., 325-6 Sunayama-cho, Naka-ku, Hamamatsu City, Shizuoka Pref., 430-8587, Japan. Hamamatsu Corp., 360 Foothill Rd., Bridgewater,NJ 08807.

[6] A. Artikov et al. [Mu2e Collaboration], Nucl. Instrum. Meth. A 890, 84 (2018).

[7] Arrhenius Equation, Encyclopedia Britannica Online, https://www.britannica.com/science/Arrhenius-equation, April 242017.

[8] R.S. Spencer and D.E. Dillon, The viscous flow of molten polystyrene, J. Colloid Sci., 3, 163-180, 1948.

[9] Serge Bourbigot, Jeffery W. Gilman, Charles A. Wilkie, Kinetic Analysis of the Thermal Degradation of Polystyrene-montmorillonite nanocomposite, June 2004.

[10] Jeffery D. Peterson, Sergey Vyazokvkin, Charles A. Wight, Kinetics of the Thermal and Thermo-Oxidative Degradation of Polystyrene, Polyethylene and Poly(propylene), 2001. 